\title{
25 Jahre UNFALLCHIRURGIE - Aufbruch ins Jahr 2000
}

D as vor Ihnen liegende Heft ist das letzte des Jahrgangs 1999. Gleichzeitig ist damit der 25. Jahrgang abgeschlossen. Dieses Jubiläum ist auch ein angemessener Anlaß, die Zeitschrift zum Millenniumswechsel für neue Herausforderungen zu stärken. Wie bereits im Editorial der UNFALLCHIRURGIE 3-4/1999 dargestellt, ist die UNFALLCHIRURGIE in jüngere Hände übergegangen mit dem Ziel, die Zeitschrift in einem europäischen Kontext neu zu etablieren.

Dies ist nun gelungen. Ab Heft 1/2000 wird die Zeitschrift UNFALLCHIRURGIE als European Journal of Trauma publiziert. Gleichzeitig ist die Zeitschrift offizielles Organ der European Trauma Society. Das derzeitige Herausgebergremium hat die Zeitschrift neu strukturiert. Die verschiedenen Bereiche der

K. H. Jungbluth

I. Marzi

J. M. Rueger

O. Trentz

Herausgeber
Unfallchirurgie werden jeweils durch Section Editors betreut, die aus verschiedenen Ländern stammen und mit der European Trauma Society für die europäische Idee stehen. Die Section Editors werden von einem breiten Advisory Board unterstützt.

Der Relaunch wird auch optisch sichtbar werden: Die Zeitschrift wird ab 2000 in einem modernen Layout, mit neugestaltetem Cover erscheinen. Parallel wird das European Journal of Trauma dann zusätzlich in einer elektronischen Ausgabe, über den Online Dienst von Springer-LINK, vorliegen.

Die Herausgeber und der Verlag möchten diese Gelegenheit auch nutzen, allen zu danken, die in den letzten Jahren die Zeitschrift mitgestaltet haben, insbesondere dem Beirat der Zeitschrift.
O. Renn

Verlag 